\title{
Spectral Transmission of Porcine Crystalline Lens at Post-Mortem Interval.
}

\author{
*Oluwasola M. Ojo ${ }^{1}$, Olajire B. Ajayi ${ }^{2}$, Mutali J. Musa. ${ }^{2}$ \\ ${ }^{1}$ Department of Optometry and Vision Science, University of Ilorin, Kwara State, Nigeria. \\ ${ }^{2}$ Department of Optometry, University of Benin, Edo State, Nigeria.
}

"Corresponding author: Oluwasola M. Ojo|E-Mail: michosola@gmail.com. | Tel: +2347032063614

\section{Abstract}

Purpose: Determining the total spectral transmission of the porcine crystalline lens at various post mortem intervals.

Method: An experimental study conducted in the Department of chemical Pharmacology, Faculty of pharmacy, University of Benin. Twenty pairs of porcine eyes were obtained from an abattoir in Benin City. The crystalline lenses were carefully extracted from the enucleated porcine eyes at various post- mortem intervals $\left(0,12,24,36\right.$ and 48hours) and their spectral transmissions were measured using a $\mathrm{T}_{80} \mathrm{UV}$-VIS Spectrophotometer. Measurement at 0 hour was used as a control being the baseline.

Result: The result showed a strong negative correlation between increased post mortem intervals and transmission of the crystalline lens for both eyes, as the post-mortem time interval increases, the transmission of long wavelengths $(\mathrm{r}=-0.992, \mathrm{P}=0.001)$, mid wavelengths $(\mathrm{r}=-0.994, \mathrm{P}=0.000)$ and short wavelengths $(r=-0.991, P=0.001)$ decreases significantly. It was also observed there was no significant difference $(\mathrm{P}>0.05)$ existed between both eyes in the different wavelength regions with increased post mortem interval.

Conclusion: The study shows that time after death has effect on the spectral transmission of crystalline lens which can serve as important information in forensic evaluation.

Key words: Post mortem interval, crystalline lens, spectral transmission, spectrophotometer and porcine.

\section{Introduction}

The post-mortem interval can be described as the time since death which is the time between the death of an animal and the period of which the body is discovered. ${ }^{1}$ Establishment of this interval provides information about the circumstances resulting to the death by giving the period during which the death may have occurred. ${ }^{2}$ However, autopsy remains the standard criteria for quality assessment in medicine which enables investigators or physicians to see 
the exact cause of death and witness the nature of changes that has occurred within the body as a result of age, diseases and lifestyle. ${ }^{3}$ In so doing, specific people or events may be excluded, or included, in the investigations that follow. Moreover, in human forensic medicine, study of the post-mortem interval has been one of the most popular topics of research but estimation of the post-mortem interval is much less developed in veterinary medicine. ${ }^{4}$

Estimating post-mortem interval (PMI) is a difficult and challenging problem in forensic medicine and especially in medico-legal investigations. ${ }^{5}$ Studies of various metabolic, physiochemical and biochemical changes from several fluid of the body such as cerebrospinal fluid, blood and vitreous humor have been used to estimate the PMI. ${ }^{6}$

Sequence of changes that take place after death, occur in a comparatively orderly fashion and several external factors and intrinsic characteristics may speed up or slow down the decomposition of the dead body. Shortly after death, postmortem changes commence and progress along a timeline. ${ }^{7}$

Alteration of the body after death occurs by two processes; putrefaction which involves the action of bacteria on tissues of the body and autolysis which is associated with breakdown of substances by endogenous substances. These two processes occur simultaneously and may be affected by environmental conditions. ${ }^{7,8}$

There are many types of changes that take place after death which can be use in determination of postmortem interval. Some of these changes are; settling of blood in the lowest-placed parts of the body, body cooling, stiffening of limbs, insect activity on the corpse and changes in eye chemistry such as in aqueous humour, vitreous humour and crystalline lens. ${ }^{9,10}$

The forensic experts have been helped immensely to ascertain the time since death (TSD) more precisely through the determination of the chemical abnormalities and various techniques have been used to estimate TSD even in present times.

The crystalline lens is surrounded by fluid anteriorly (aqueous humor) and posteriorly (vitreous humor) and it is situated to the rear of the iris. About 10003000 strata of fiber cells are found in the lens and the proper arrangement of these fiber cells determines the transparency of the lens. ${ }^{11}$ Another very important component that helps to maintain the transparency of the crystalline lens is $\alpha$ crystalline which is about $90 \%$ of the total protein content and $35 \%$ of the net weight of the lens. ${ }^{12}$ Despite the fact that the crystalline lens has no blood vessels, being an avascular structure, physical and chemical changes take place after the death, whether the eye is left intact in the animal or the lens is excised from the globe. ${ }^{13}$ However, since the lens is protected between the vitreous humour and the aqueous humour inside the eyeball, postmortem changes are assumed to start later in the lens. ${ }^{14}$

It is also an established fact from previous studies that

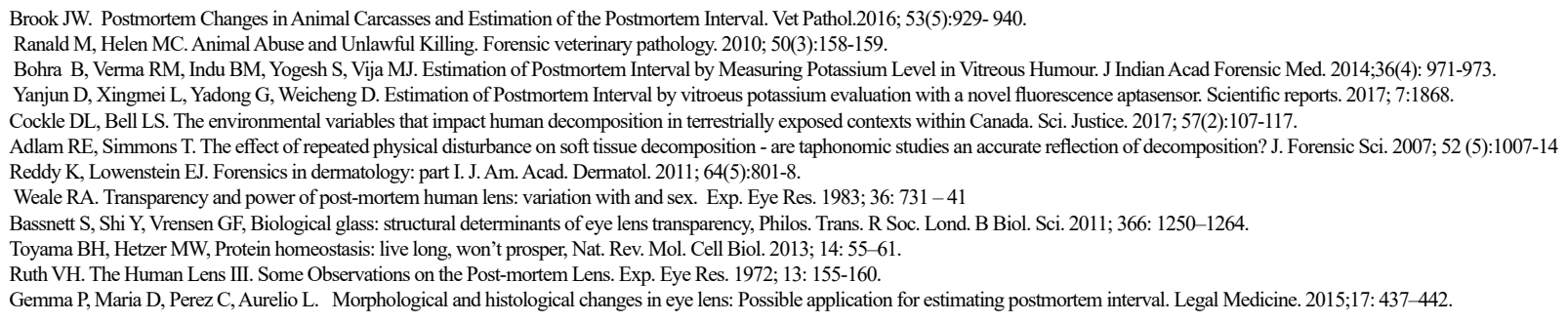


the human crystalline lens develops yellow colour with age, even loses its transmission property after death. ${ }^{15}$ However, the experimental measurements that determine the period and show the gradual loss in lens spectral transmission and the proportion of the loss in its transmission attribute are very few.

The aim of this study was to determine the total spectral transmission of the pig crystalline lens at various post mortem intervals in order to give approximately the range of time of death which would help in forensic investigation.

Also, changes in lens spectral transmisssion at postmortem could be a useful starting point for testing the possibility of present visual impairment conditions in a cadaver at the time of death. ${ }^{14}$ Furthermore evaluation of the lens would provide the added benefit of associating different stages of cataracts with accidents providing additional information relevant to the development of preventive measures. ${ }^{16}$

In conclusion, the post-mortem determination of spectral transmission of crystalline lens would provide helpful information that could be used during legal proceedings and it would be a good complement to clinical data in cases where there is no medical documentation.

\section{Methods}

This is an experimental study conducted in the Department of chemical Pharmacology, Faculty of pharmacy, University of Benin. A total of 40 eyes were used for this study which comprised 20 pairs of enucleated porcine eyes obtained from a nearby abattoir in Benin City and were classified into 4 groups of 5 pairs each.
The crystalline lenses of enucleated eyeballs of the first group were extracted within an hour after death in order to accurately predict the baseline of spectral transmission of the crystalline lens as at the time of death (0hr).

The enucleated eyeballs were separated into the right eyes and the left eyes in different tagged sample carrier indicating right or left eyes and were transported to Chemical pharmacology laboratory Department. Immediately after the eyes were transported to the laboratory, the spectral transmissions of 5 pairs of crystalline lenses were measured for the first group at $0 \mathrm{hr}$. Thereafter the same procedure was done for the remaining groups at every 12 hours interval (second group 12 hours post-mortem, third group 24 hours post-mortem, fourth group 36 hours post-mortem and fifth group 48 port-mortem).

However, all the eyes used belonged to genetically similar pigs of about six months old that were fed the same diet and were slaughtered at the same age; therefore, the study was made up of a sample of homogeneous lenses. Particular care was taken in the lens removal process when detaching the lenses from the zonules to avoid damaging the capsules and carefully rinsed in distil water to remove any adhering substance such as aqueous humour or blood. Afterward, the lenses were carefully positioned in the cuvettes containing saline solution placed in the spectrophotometer after the instrument had been zeroed using saline solution as a reference to measure the transmittance. Earlier studies had shown that if the material was refrigerated, no significant change in either the image forming properties of the lens or in its transmissivity could be detected. ${ }^{7,8}$ However,

Cockle DL, Bell LS. The environmental variables that impact human decomposition in terrestrially exposed contexts within Canada. Sci. Justice. 2017; 57(2):107-117

Adlam RE, Simmons T. The effect of repeated physical disturbance on soft tissue decomposition - are taphonomic studies an accurate reflection of decomposition? J. Forensic Sci. 2007; 52 (5):1007-14 Artigas JM, Felipe A, Navea A, Adriana F, Artigas C. Spectral Transmission of the human crystalline lens in adult and elderly persons: color and total transmission of visible light. Investigative Ophthalmology and visual science. 2012; 4076-4084.

Stemberga V, Petaros A, Kovacevic D, Coklo M, Simicevic N, Bosnar A. The assessment of lens opacity postmortem and its implication in forensics. J.Forensic Leg. Med. 2013;20: 1142-1144. 
the eyes were neither refrigerated nor stored in any solution before the lenses were excised for their transmittance to be measured. No time was wasted between the excision and spectrophotometric measurement so as to minimize the effects of cold cataract, and to allow the disappearance of effects due to occasional minor reversible trauma resulting even from the most careful handling. All the animals were examined for any form of illness that could affect the general health, hence having a negative effect on the readings gotten from the samples.

\section{Spectral Transmission Measurements:}

The curves for transmission were obtained by using a T80 Double Beam UV/VIS spectrophotometer (PG Instruments, Alma Park, Wibtoft, Leicestershire, LE17 5BH, United-Kingdom) which is capable of measuring in the range of 200-800 $\mathrm{nm}$. The manufacturers' instructions were followed as regards determining the baseline of the instrument; also attention was drawn to the possibility of backlash in the control of the wavelength settings. During the measurement, automatic recorder was not used, because it may lead to some systematic errors being undetected, ${ }^{10}$ however, the instrument was zeroed after each reading had been taken.
This was done to avoid changing in transmittance resulting from the exposure to some of the ultraviolet measuring wavelengths. Accordingly, wavelengths between $200 \mathrm{~nm}$ to $800 \mathrm{~nm}$ were set manually and spectrophotometer gave readings for transmittance of the sample materials as whole process was repeated for each sample and results printed.

Data obtained from the experiment was analysed using Statistical Package for Social Science (SPSS) version 22. Pearson Correlation Test was used to determine the correlation between the spectral transmission of Crystalline Lenses and Postmortem interval with the significance level set to be $\mathrm{p}<0.05$.

\section{Results}

The table 1 below shows the range of transmission (\%) of the wavelengths recorded in this study. These were grouped according to the length of the wavelength in the visible spectrum-long wavelengths (representing the red and orange region of the spectrum), mid wavelengths (representing the green and yellow region of the spectrum) and short wavelengths (representing the blue violet region of the spectrum).

Table 1: Range of transmittance recorded at the different post-mortem time interval.

\begin{tabular}{|c|c|c|c|c|}
\hline Time (hours) & Eye & $\begin{array}{c}\text { Long } \\
780-590 \mathrm{~nm}(\%)\end{array}$ & $\begin{array}{c}\text { Mid } \\
570-490 \mathrm{~nm}(\%)\end{array}$ & $\begin{array}{c}\text { Short } \\
480-380 \mathrm{~nm}(\%)\end{array}$ \\
\hline \multirow[t]{2}{*}{0} & $\mathrm{R}$ & 92 & 85 & 73 \\
\hline & $\mathrm{L}$ & 92 & 84 & 73 \\
\hline \multirow[t]{2}{*}{12} & $\mathrm{R}$ & 84 & 78 & 52 \\
\hline & $\mathrm{L}$ & 78 & 74 & 64 \\
\hline \multirow[t]{2}{*}{24} & $\mathrm{R}$ & 66 & 64 & 41 \\
\hline & $\mathrm{L}$ & 62 & 60 & 45 \\
\hline \multirow[t]{2}{*}{36} & $\mathrm{R}$ & 53 & 45 & 28 \\
\hline & $\mathrm{L}$ & 52 & 47 & 34 \\
\hline \multirow[t]{2}{*}{48} & $\mathrm{R}$ & 30 & 28 & 16 \\
\hline & $\mathrm{L}$ & 31 & 27 & 16 \\
\hline
\end{tabular}

$R=$ Right eye, $L=$ Left eye 
The line charts shown in fig. 1 and 2 represent the average transmission of the wavelengths at the various time interval for the right and left eye respectively. The long wavelengths had the highest amount (\%) of transmission while the short wavelengths had the least across the various time intervals for both eyes.

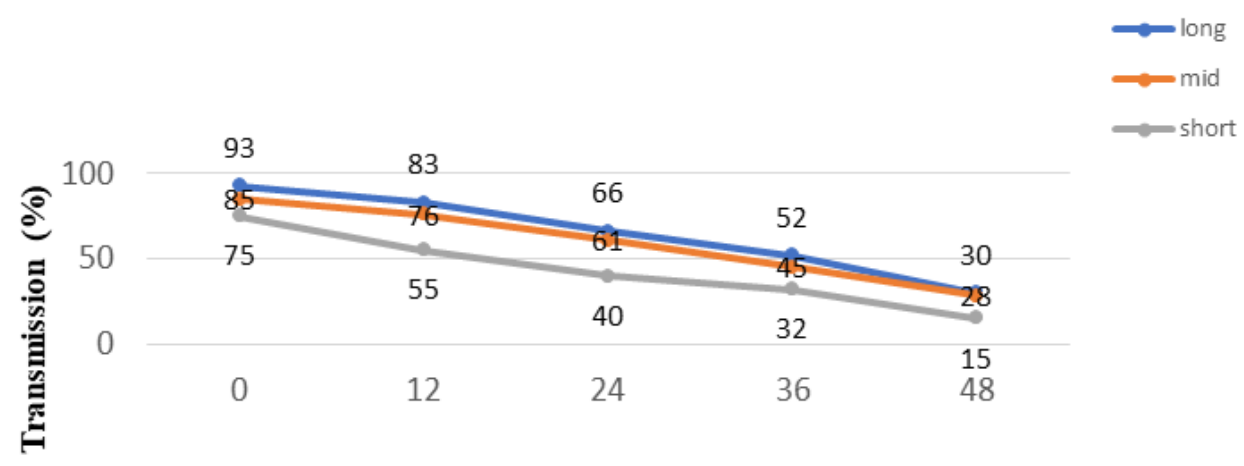

Post-mortem time interval (hours)

Figure 1: Line chart showing the average transmittance of the different wavelength groups at the different post-mortem time interval (right eye).

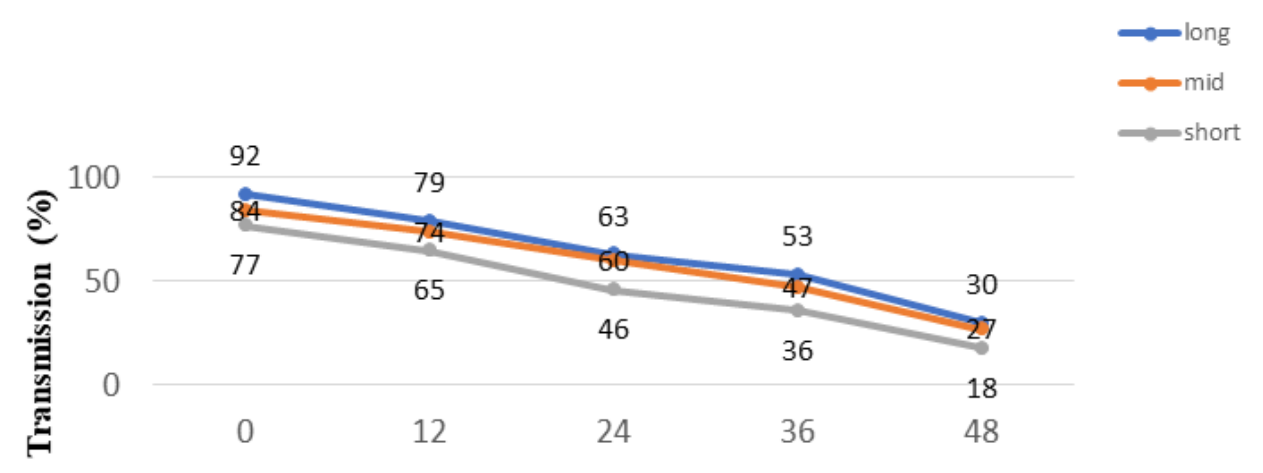

Post-mortem time interval (hours)

Figure 2: Line chart showing the average transmittance of the different wavelength groups at the different post-mortem time interval (left eye).

Via Pearson's correlation, the relationship between the post-mortem time and the transmission at the different regions was determined. The regression plots as well as the correlation coefficients for both eyes (right and left) are shown in fig. 3 and fig.4 respectively.

For the right eye, as the post-mortem time interval increases, the transmission of long wavelengths $(r=$ -0.992, $P=0.001)$, mid wavelengths $(r=-0.994, P=0.000)$ and short wavelengths $(r=-0.991, P=0.001)$ decreases significantly. Likewise, for the left eye, the increase in post-mortem time interval resulted to significant decrease in the transmission of long wavelengths $(r=-0.992, P=0.001)$, mid wavelengths $(r$ $=-0.993, P=0.001)$ and short wavelengths $(r=-0.996, P=0.000)$. 


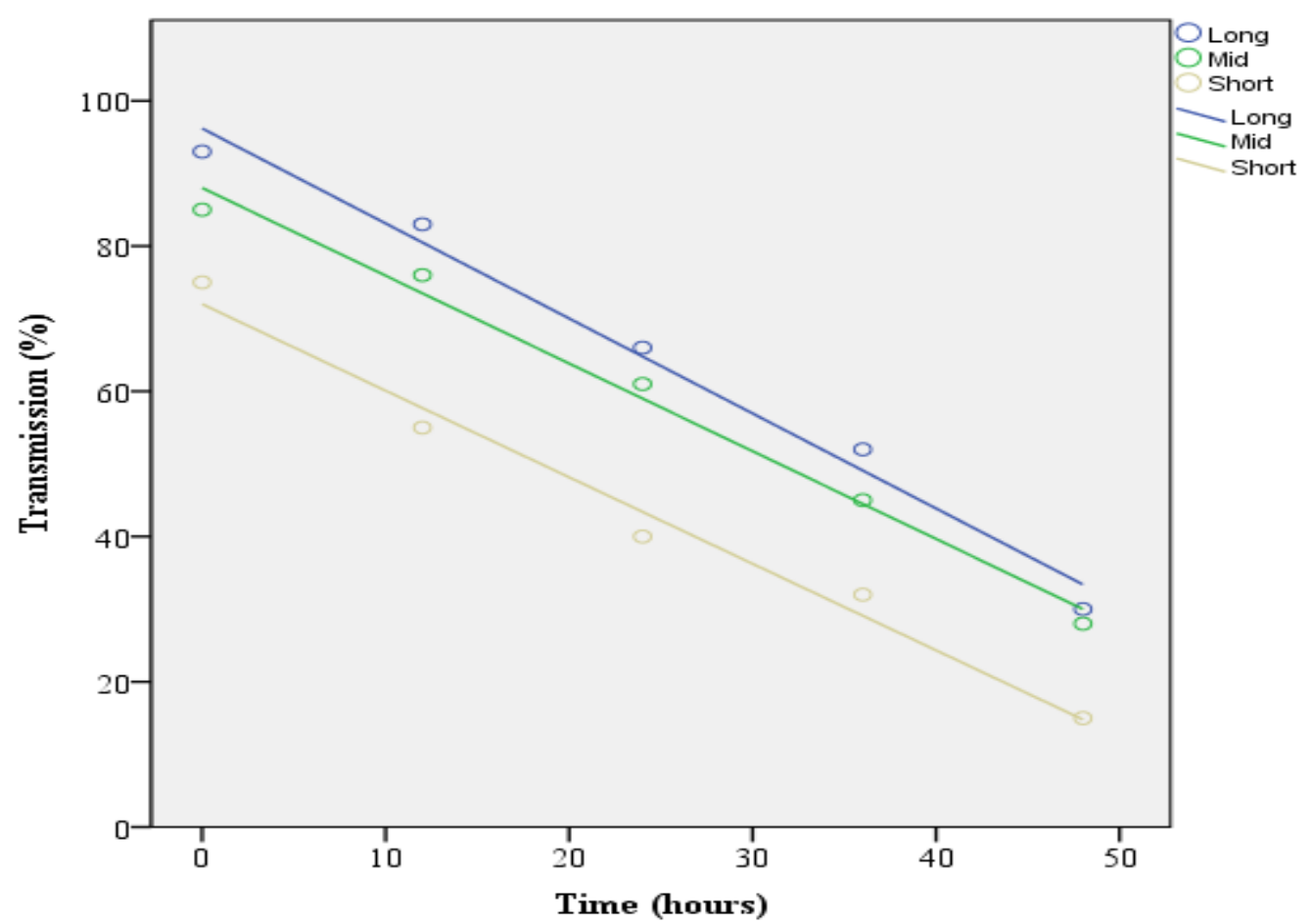

Figure 3: Regression plot showing a strong negative relationship between time and the transmission in the different regions (right eye).

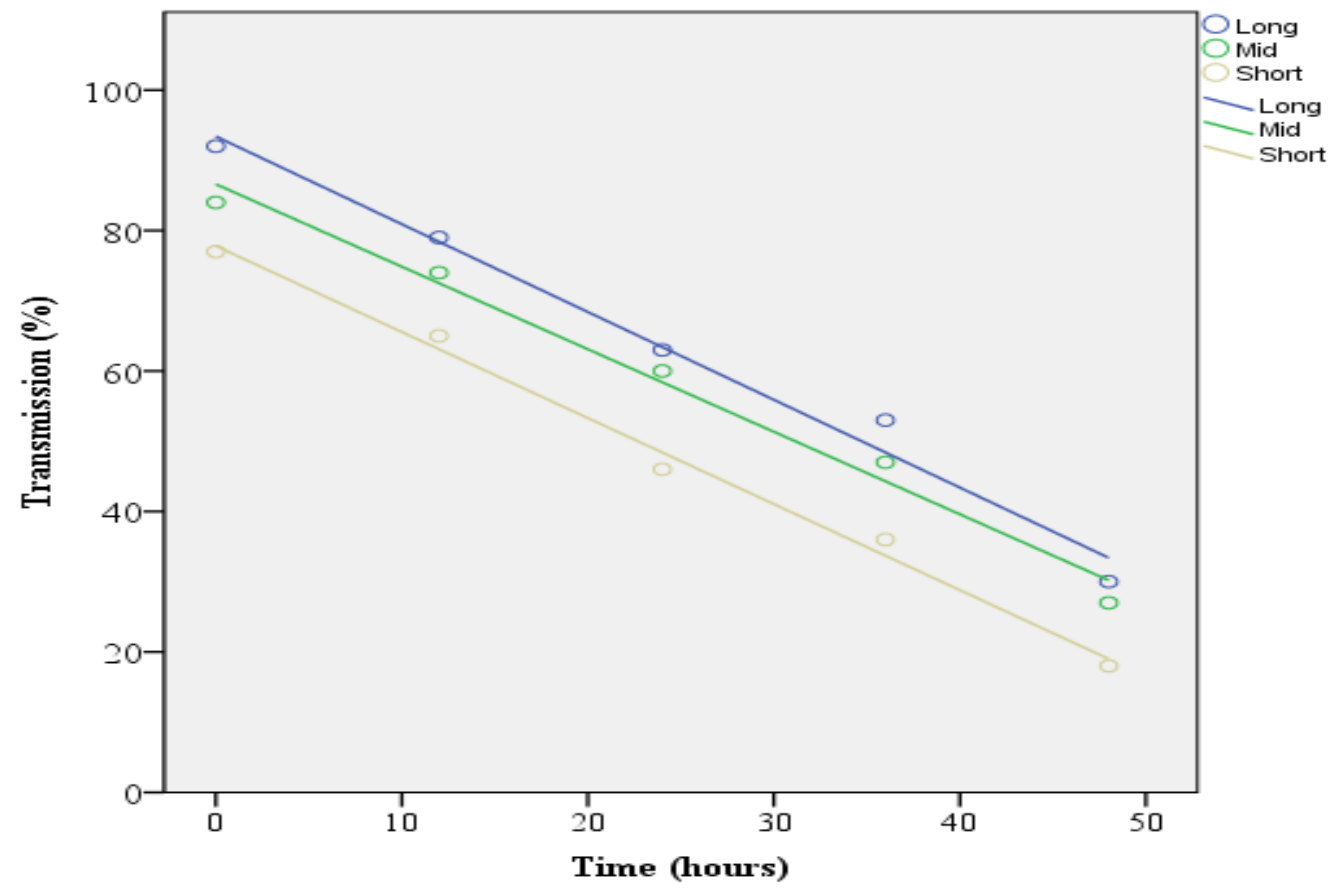

Figure 4: Regression plot showing a strong negative relationship between time and the transmission in the different regions (left eye). 
Table 2: comparison between the long wavelengths transmission of the right and left porcine lens at increasing post-mortem interval (PMI)

\begin{tabular}{ccc}
\hline Time [hours] & Right $[\mathrm{T}] \%$ & Left $[\mathrm{T}] \%$ \\
\hline 0 & 93 & 93 \\
12 & 84 & 79 \\
24 & 66 & 62 \\
36 & 53 & 52 \\
48 & 30 & 31 \\
\hline
\end{tabular}

Using the independent sample T-test, there was no significant difference $(\mathrm{P}>0.05)$ between the lenses in the left and right eye in the transmission of long wavelengths $(P=0.930)$.

Table 3: comparison between the medium wavelengths transmission of the right and left porcine lens at increasing post-mortem interval (PMI)

\begin{tabular}{ccc}
\hline Time [hours] & Right $[\mathrm{T}] \%$ & Left $[\mathrm{T}] \%$ \\
\hline 0 & 85 & 84 \\
12 & 78 & 74 \\
24 & 64 & 60 \\
36 & 45 & 47 \\
48 & 28 & 27 \\
\hline
\end{tabular}

Using the independent sample T-test, there was no significant difference $(\mathrm{P}>0.05)$ between the lenses in the left and right eye in the transmission of mid wavelengths $(P=0.968)$

Table 4: Shows comparison between the short wavelengths transmission of the right and left porcine lens at increasing post-mortem interval (PMI)

\begin{tabular}{ccc}
\hline Time $[$ hours $]$ & Right $[\mathrm{T}] \%$ & Left $[\mathrm{T}] \%$ \\
\hline 0 & 74 & 75 \\
12 & 53 & 64 \\
24 & 41 & 44 \\
36 & 27 & 34 \\
48 & 16 & 16 \\
\hline
\end{tabular}

Using the independent sample T-test, there was no significant difference $(\mathrm{P}>0.05)$ between the lenses in the left and right eye in the transmission of short wavelengths $(P=0.741)$. 


\section{Discussion}

Over the years, the post-mortem changes have been studied using various techniques. Yet the measurement of the estimation of post-mortem interval using spectral transmission of ocular media has not been studied intensively. Hence, in this study, the spectral transmission of porcine crystalline lenses were measured at various post-mortem intervals at room temperature $\left(25^{\circ} \mathrm{c}\right)$ to ascertain if there is a significant correlation between the time after death and spectral transmission of porcine lens within long, medium and short wavelength of the visible spectrum. The transmission measurements were taken at 0hour, 12hours, 24hours, 36hours and 48hours after death.

As shown in fig. 1 and 2, the spectral transmission at Ohour after death showed a maximum transmission along a long wavelength which was about $95 \%$ and a reduction in the transmission of short wavelength. This was supported by a study carried out by Artigas et al. ${ }^{15}$ They found out that lens becomes more selective and filters short wavelengths more than long wavelengths as a transmission curves becomes lower at a certain period. This is also in agreement with the findings of Said and Weale on the direct transmission of lens in the living eye.

At post-mortem interval of 12 hours, as shown in the figure 3 and 4 , there was a slight reduction in the transmission of long waves (both left and right), concomitantly a marked reduction in the short wavelengths as compared with transmission at Ohour. This was also supported by Artigas, 2011, he found out that pig lens transmission reduced at UV A region which eventually disappeared as time goes on after death. ${ }^{17}$
At post-mortem interval of 24 hours, there was a marked reduction in the transmission of both long and medium wavelength with no significant different between them. But the short wavelength transmission showed a significant reduction when compared with both long and medium wavelengths. At post-mortem interval of 36 and 48hours the spectral transmission along the long, medium and short wavelength of the visible spectrum has been drastically reduced with minimum reduction occurred at short wavelength.

When the data obtained was statistically analysed using SPSS Version 22 with the Pearson's correlation, the analysis showed that there was a strong negative correlation between the postmortem interval and spectral transmission of the porcine lens. That is as post-mortem interval increased the spectral transmission of the porcine lens decreased for both right and left eyes.

The reduction of the lens transmission at postmortem interval may not be far from the fact that shortly after death the cornea begins to lose its sodium ions content, thereby increases the aqueous humour sodium concentration which eventually leads to increase in the concentration of sodium in the lens. As a result of change in sodium concentration cum with the influx of water and chloride from aqueous and vitreous humour, the optical density of the crystalline lens increases which lead to decrease in its spectral transmission. This is also supported by Beer-Lambert law which states that the amount of energy absorbed or transmitted by a solution is proportional to the solutions molar absorptivity and concentration of solute. According to Ruth van et $\mathrm{al}^{7}{ }^{7}$ it was stated that increased in sodium

Cockle DL, Bell LS. The environmental variables that impact human decomposition in terrestrially exposed contexts within Canada. Sci. Justice. 2017; 57(2):107-117. Artigas JM, Felipe A, Navea A, Adriana F, Artigas C. Spectral Transmission of the human crystalline lens in adult and elderly persons: color and total transmission of visible light. Investigative Ophthalmology and visual science. 2012; 4076-4084.

Artigas JM, Felipe A, Navea A, Artigas C, Garcia-Domene MC. Spectral transmittance of intraocular lenses under natural and artificial illumination: criteria analysis for choosing a suitable filter. Ophthalmology.2011;118: 3-8. 
concentration in post mortem lens is presumably a supplementary cause may be the postmortem as a result of gradual cessation of metabolism increase in concentration of sodium in the aqueous in the lens remaining in the eye after death but humour battling the crystalline lens.

\section{Conclusion:}

Comparing the spectral transmission of right and left porcine lenses with increase in post-mortem interval at room temperature $\left(25^{\circ} \mathrm{c}\right)$, it was observed no significant difference $(\mathrm{P}>0.05)$ existed between both eyes in the different wavelength regions.

[How to cite this article: Ojo OM, Ajayi OB, Musa MJ. Spectral Transmission of Porcine Crystalline Lens at Post-Mortem Interval.Journal of the Nigerian Optometric Association. 2020;22(1): 35-43 ] 